\title{
CHOOSING WISELY
}

Luis C L Correia*, Luisa Santos Pereira**, Fernanda Lopes***

Corresponding author: ondence: Luís Cláudio Lemos Correia lccorreia@terra.com.br

* PhD, Full professor at Bahiana School of Medicine and Public Health

** Medicine undergraduate student at Bahiana School of Medicine and Human Health

*** Medicine undergraduate student at Bahiana School of Medicine and Human Health

In 2012, the American Board of Internal Medicine initiated the Choosing Wisely campaign in the United States, which today has officially expanded to other countries, such as Canada, England, Germany, Italy, Netherlands, Switzerland, Australia, New Zealand and Japan. These countries are grouped together into what is called the Choosing Wisely International. This initiative serves as an inspiration to any country that insists on imitating the American standard of pseudo-scientific resource consumption. Brazil is one of them.

The Choosing Wisely initiative arises from the perception that there is lack of wisdom in the exaggerated or inappropriate use of resources in health care. Choosing Wisely is a campaign that goes together with the "less is more" paradigm.

It would be imposing and unwelcome if the American Board of Internal Medicine started a campaign against conducts that are usually adopted by the medical specialties. Thus, the responsibility of self-criticism was given to the specialists, instead of simply criticizing them. Therefore, specialties were solicited to point out currently used medical conducts that should not be adopted. This forced specialists to reflect and counter-indicate their own futile conducts.

Another aspect that was emphasized by the organizers is that the Choosing Wisely recommendations do not have the primary aim of reducing costs, but rather to improve the quality of care, which must be based on evidence, increasing the probability of benefit and reducing the risk of harm to the health of individuals.

Furthermore, taking into consideration our present moment, it is important to emphasize that this is not a governmental initiative in these countries - on the contrary, it is an initiative of the medical society itself.

Choosing Wisely recommends what we should not do. It brings an interesting paradigm, since we are usually trained to discuss what we should do. The guidelines say more about what we should do, rather than what we should not do. And the recommendations of what not to do (Class III recommendations) are normally limited to conducts with proven harm. However, besides the proof of harm, there are other reasons why we should not use certain conducts. To put it in another way, it does not mean that we should do something simply because it is not harmful.

The burden of proof is in the performance (efficacy) and utility (relevance) of a given conduct. This way, the following reasons can justify not using certain conducts:

1. Harmful therapy - this is obvious, therefore this is not the primary focus of Choosing Wisely. 
2. Therapy with unknown efficacy (not yet demonstrated) - there are several examples of conducts that cannot be classified as extremely plausible, but that are systemically adopted based on beliefs.

3. Therapy with proven inefficacy, but safe - this is also done, since clinical trials with negative results are commonly not valued for going against our beliefs.

4. Diagnostic or prognostic tests that are applied in useless situations (futile), bringing results that are potentially harmful (overdiagnosis).

The north-american "Choosing Wisely" site brings interesting recommendations from each specialty and should be visited by everyone with a reflexive attitude. This is a vanguard thought, which combats the paradigm that was previously mentioned, the "active doctor mentality". Although this paradigm has been extremely prevalent, however, it will probably become obsolete within the next 10 to 20 years. It is up to each one of us to decide which path to choose: a reflexive path, in the vanguard, or the traditional and outmoded path.

In the context of cardiology, the campaign was very coherent when it stated "we should not search for myocardial ischemia in asymptomatic patients". Observe how (on average) what physicians do is exactly the opposite. The reality is that a person cannot even pass by a cardiologic clinic without obtaining a non-invasive exam solicitation for the search of coronary disease (usually a cardiac stress test, but also myocardial scintigraphy is frequently requested, and the coronary tomography also emerges as an accurate and potentially useful method, but with the risk of being used to promote overdiagnosis). Finally, Choosing Wisely says, "please, do not search for coronary disease in the pre-surgical assessment of low-risk surgeries." This is how smart people think.
We also like the recommendation for orthopedists to not prescribe chondroitin or glucosamine (Condroflex) for the treatment of knee arthritis. Yes, it is that medication that so many patients affirm to have reduced their knee pain, but we know that it is a result of the placebo effect, very well demonstrated by a large randomized clinical trial published in the New England Journal of Medicine. There are also those who say that if the pain improves, it does not matter if it is secondary to the placebo effect. However, should we, routinely, propose a medicine based on fantasy? Where might we end up?

Once, one of the leaders of the Choosing Wisely in the United States compared this initiative to an Indiana Jones movie, where the hero searches for the Holy Grail. In the final scene, there are many goblets and only one chance of choosing the correct goblet, that which would be the Holy Grail. The guardian of the goblets warns the characters: Choose Wisely. The first to choose, in an obvious manner, chooses the most beautiful and precious goblet. However, as we know, in science, the plausible choice is not always the true one. The chosen goblet was not the Holy Grail and the villain fails, and ends up turning into a skeleton. On the other hand, Indiana Jones is a scientist, and he uses his scientific mind to make the wisest choice. He chooses the simpler goblet, which is more coherent to the values of Jesus Christ. He ends up choosing correctly, conquering the Holy Grail.

As physicians, we must think wisely. Using resources without any scientific proof or in an exaggerated manner approximates us to the villain in the movie and distances us from Indiana Jones, our hero. Being a hero is not about using the active mentality, suggesting futile or uncertain procedures, exams, or treatments. Being a hero is to know when not to act, assuming our own uncertainties, and alternating with moments that require a more active attitude. 